\title{
Effectiveness of Cognitive Behavior Therapy (CBT) for Chronic Low Back Pain (CLBP): A Systematic Review
}

\author{
Nur Melizza \\ Magister Student of Nursing, Faculty of Nursing Universitas \\ Airlangga, Surabaya, Indonesia \\ nur.melizza-2016@fkp.unair.ac.id
}

\author{
Ulum Mabruroh \\ Magister Student of Nursing, Faculty of Nursing Universitas \\ Airlangga, Surabaya, Indonesia \\ ulum.mabruroh-2016@fkp.unair.ac.id,
}

\begin{abstract}
Nonspecific chronic low back pain (CLBP) is a main cause of activity limitations, absenteeism, and high health care expenses. The prevalence of CLBP is estimated at approximately $23 \%$, and activity limitation due to low back pain had been found in $11 \%$ to $12 \%$ of the population. Low back pain is consistently among the top six most costly health problem and, accounting for incidence, one of the top three most disabling conditions in developed countries. Psychosocial contributing to this condition is felt significantly, and Cognitive Behavioral Therapy (CBT) offers a more effective treatment and potentially more economical. The purpose of this study was to evaluate the effectiveness of Cognitive Behavioral Therapy as an intervention to overcome Chronic Low Back Pain. The key word to database search was "Cognitive Behavioral Therapy, Chronic Low Back Pain, Chronic Pain, and Cognitive Therapy". According to the journal article that already found and reviewed from Google Scholar, PubMed, and Science Direct database with will date restriction started from 2007 until 2015 showed that CBT could be an intervention to increase physical activity, decrease pain, and disability. CBT can be used as an intervention in CLBP patients because it could increase physic activity, decrease pain, and lessening disability also spent least cost.
\end{abstract}

Keywords-Cognitive Behavioral Therapy, Chronic Low Back Pain, Chronic Pain, Cognitive Therapy

\section{INTRODUCTION}

Low back pain is one of the most prevalent and expensive musculoskeletal conditions. Non-specific chronic low back pain does not have a defined source, 78 and the pathogenesis is not entirely understood. In the absence of a peripheral pathology, central sensitization has been hypothesized to explain the development and maintenance of non-specific chronic low back pain. Primary mechanisms is an increased of excitatory neurotransmitters release at spinal level, influencing pain perception via the spino-thalamic pathway and altered top-down pain control from the brain[1].

Ref [2] explained that in a large epidemiological survey on the prevalence and impact of chronic pain in Singapore found that $8.7 \%$ of the total population suffering from chronic pain. Among this population, the major cause of pain was musculoskeletal and the main locations of pain were knee, shoulder, and lower back. More than half reported that pain affected their daily activity that required lower limb activities such as the ability to lift objects (58\%), walk (52\%) and exercise $(50 \%)$. In fact, $38.6 \%$ reported difficulty functioning in their normal roles. Hence, we find a significant impact on work and daily function of those with chronic NSLBP in Singapore resulting in disability as reported.

Back pain occurs in most individuals and is one of the most common reasons for health care seeking [3]. The annual costs of medical care for back pain in the United States have been estimated approximately $\$ 25$ billion[4], and productivity losses at $\$ 28$ billion[5]. Back pain does not a typically disease that resolve within a few weeks completely as previously believed. Rather, recurrent and chronic back pain are common[6]-[8]. Recurrent or chronic back pain frequently result in significant worry and interference with activities [7].

Chronic nonspecific low back pain (CLBP) is a major cause of activity limitations, absenteeism, and high health care expenses. The prevalence of CLBP is estimated at approximately $23 \%$, and activity limitations due to low back pain have been found in $11 \%$ to $12 \%$ of the population. Low back pain is consistently among the top six most costly health problem and, accounting for incidence, one of the top three most disabling conditions in developed countries. International guidance recommends that people with persistent non-specific low back pain remain active physically[9]

Compared with advice to remain active, physical treatments (structured exercise, acupuncture, manipulation, and postural approaches) produce small to moderate mean short-term ( $\leq 4$ months) benefits, but typically small or nonsignificant mean longer-term ( $\geq 12$ months) benefits. There is some proof of concept to support cognitive behavioral interventions, but trials that tracked response beyond 6 months reported mixed results[10].

Cognitive Behavioral Therapy (CBT) is a psychotherapeutic approach that is frequently applied and most have been proven effective in treating a variety of disorders. The basic principles of CBT is that the way we think in certain situations affects how we feel emotionally and physically, and change our behavior, CBT also offer a potential treatment modality more economical. 


\section{METHODS}

This review production started with found journal article with PICO framework, researched population was patient CLBP, intervention which did by research was CBT, intervention comparison was by another therapy for CLBP, and the result of research was increase physic activity, pain decrease, disability CLBP. The key word was "Cognitive Behavioral Therapy", or "Cognitive therapy" and "Chronic Low Back Pain", or "Chronic Pain" in the database Google Scholar, PubMed, and Science Direct with date restriction started from 2007 until 2015.

From the searching process we found 32 journals, and 13 journals selected which fulfilled inclusive criteria: patients with LBP more than 3 months. Then we did review from journal selected.

\section{RESULT}

\section{A. Cognitive Treatment in a Changing Perceptions of Disease Patients}

According to result of study as in [10] showed that there was significant difference statistically between intervention group and control group after 18 weeks intervention, there was alteration in patients physical activity, that happen relevant alteration on clinic $(19,1 \mathrm{~mm})$ for intervention group. The difference significant statistic also found on most scale for illness perception.

Cognitive Treatment of Illness Perceptions (CTIP) consist of 10 until 14 session an hour individual self-care and will give every weeks for every patient from CTIP group by experience physic therapy or occupation therapy that appropriate with protocol therapy. CTIP was conducted in four phase start from perception mapping patient about disease, could be called perception resist patient who have maladaptive about diseases, perception changes which lead to increase physical activity and the last examine alternative perception and with the strong confirmation used for daily practice.

Decrease 18 until $24 \mathrm{~mm}$ for Patient Specific Complains (PSC) certainly as clinic alteration which relevant for patient with low back pain. The measure of sample counted by minimal alteration $18 \mathrm{~mm}, 2$-ided alpha from 0,05 , a 1 - beta from 0,90, and standard derivation 26,01. This standard derivation measured from PSC data which available from patient CLBP in the same central which in side from this study. For understanding the side effect of group from alteration on the secondary result, we measured the Illness perception using Illness Perceptions Questionnaire-Revised (IPQ-R) and Quebec Back Pain Disability Scale (QBPDS) identical analysis which did as like PSC. First analysis covariances on difference scale IPQ-R and QBPDS. Incomplete analysis consist of covariances which appropriate with first score from IPQ-R or QBPDS who include as covariate, feedback score as variable depended and group as variable independence. For analyses appropriate covariate identical used for measure main result assumption for linear regression analysis will be check for all analyses. There is no discovery for infringement

\section{B. Cognitive Behavioral Therapy and Cost-Effectiveness}

According to result of research by[10], intervention CBT consist of 90 minutes early self assessment and six session 90 minutes from CBT group, which targeted behavioral and conviction about physical activity. Every session CBT group include average 8 subject and every session guided physiotherapy, nurse, psychologist or occupation therapy who have received coaching for 2 day who giving intervention CBT.

The score principle in questioner Roland Morris Back Pain Disability and Modified Von-Korrf Scale, which separate measure pain and disability. This result measure by means of questioner. Secondary result related of spirit quality and Physical Health-related Quality of Life Scores from 12-item health survey short-form (SF-12), fear-avoidance beliefs questionnaire scores, and pain self-efficacy scale scores. The cost, include directly intervention and all of the cost another caused back pain.

The research principle was random patient, mean duration back pain (early first onset) did 13 years. On 12 month, patient who receive intervention CBT group had mean 2,4 point increase in Roland Morris score just than mean increase 1,1 point among patient with usual care $(\mathrm{P}<0,001)$. Score pain and disability Von Korrf too increase with more sum with CBT group than usual care: for pain, $13,4 \%$ vs $6,4 \%$ ( $\mathrm{P}<0,001$ ); for disability $13,8 \%$ vs $5,4 \%$ ( $\mathrm{P}<0,001)$. The comparison with usual care, CBT group related too increase significant statistic better than belief, anxiety-avoidance, pain self-efficacy, and physic score SF-12 on 12 month; however, there was no significant difference between research group in score SF-12 spirit scale. Additional cost calculation qualityadjusted life-year (QALY) related with CBT was $£ 1.768$ (\$ 2.650).

On the matter supported was result the research by Johnson REI, et al., 2007, which the intervention could be influence to decrease pain scale $(-3,6 \mathrm{~mm} ; 95 \%$ confidence interval, the $-8,5,1,2 \mathrm{~mm})$ and disability $(-0,6 \mathrm{sk}$ or; $95 \%$ confidence interval, -1,6, 0,4) although small influence, however the intervention low cost with extra ratio costeffectiveness 5000 pound (U.S. \$8650) quality-adjusted lifeyear (QALY). Beside that intervention group explain to preference for that intervention and decrease significant about pain and disability.

\section{Cognitive Behavioral Therapy on CLBP}

According to result of research by [10], explain that participant more intervention group than control group which satisfied with their treatment on 12 month. Fear avoidance, pain self-efficacy, and SF-12 physical scores improve as substantial on group intervention CBT therapy not control group. This research by Marcus Beasley, 2015, also explain that a short program tCBT for patient with CWP effective for long phase and more cost effective. Although with another research by[11] explained that intervention with CBT could be good influence to increase physic activity, pain decrease or patient decrease disability with CLBP. The more effect from intervention CBT was increase live quality patient CLBP. 


\section{CONCLUSION}

CLBP was their effect from activity disturbance, limitedness physic, disability, musculoskeletal pain disturbance, and effect of live quality that already diagnostic. Various of action could be do for overcoming the problem, but another problem which coming is connecting with cost of handling and therapy. Most journal article that reviewed by researcher showed that CBT was one intervention that can be used to treat CLBP which effective to increase physic activity, pain decrease and disability, and can increase quality of life with cost effectiveness.

The summary of this study was CBT focus on pain management and activity, with variation time duration started from do minutes every session, Tuesday 1-3 months, 30 minutes 1 hours, 2 hours, 5 hours, until 10 hours in the frame time some weeks and months. In the some cost research which used was relative cheap because activity could did individual and the place was cozy, such as mean local government clinic (PUSKESMAS) or primer health services, so it could not complicated patient

\section{References}

[1] A. F. Mannion, J. I. Brox, C. T. Jeremy, and Fairbank, "Comparison of spinal fusion and nonoperative treatment in patients with chronic low back pain: long-term follow-up of three randomized controlled trials," Spine J., vol. 13, p. 1438-1448., 2013.
[2] A. J. Devasahayam, C. K. S. Lim, M. R. Goh, J. P. L. You, and P. Y. Pua, "Delivering a Back School Programme with a Cognitive Behavioural Modification: A Randomised Pilot Trial on Patients with Chronic Nonspecific Low Back Pain and Functional Disability," Proc. Singapore Healthc., vol. 23, no. 3, pp. 218-225, 2014.

[3] B. K. Cypress, "Characteristics of physician visits for back symptoms: a national perspective,” Am. J. Public Health, vol. 73, pp. 389-395, 1983.

[4] J. Frymoyer and W. Cats-Baril, "An overview of the incidences and costs of low back pain," Orthop Clin North Am., vol. 22, no. 2, pp. 263 71, 1991.

[5] J. Rizzo and et al, "The Labor Productivity Effects of Chronic Backache in the United States," Med Care, vol. 36, no. 10, pp. 1471-1488, 1998.

[6] M. Roland and R. Morris, "A Study of the Natural History of Back Pain: Part I: Development of a Reliable and Sensitive Measure of Disability in Low-Back Pain," Spine J., 1983.

[7] M. Von Korff, J. Ormel, F. Keefe, and S. Dworkin, "Grading the severity of chronic pain," Pain PubMed, vol. 50, no. 2, pp. 133-149, 1992.

[8] M. Von-Korff and K. Saunders, "The course of back pain in primary care," Spine J. PubMed, vol. 21, no. 24, pp. 2833-7, 1996.

[9] B. W. Koes, M. van Tulder, C.-W. C. Lin, L. G. Macedo, J. McAuley, and C. Maher, "An updated overview of clinical guidelines for the management of non-specific low back pain in primary care," Eur. Spine J., vol. 19, no. 12, p. 2075-2094., 2010.

[10] S. E. Lamb, Z. Hansen, R. Lall, E. Castelnuovo, E. J. Withers, V. Nichols, R. Potter, and M. R. Underwood, "Group cognitive behavioural treatment for low-back pain in primary care: a randomised controlled trial and cost-effectiveness analysis," Lancet, vol. 375, no. 9718, pp. 916-923, 2010.

[11] D. . Thompsona, J. A. Oldham, and S. R. Woby, "Does adding cognitive-behavioural physiotherapy to exercise improve outcome in patients with chronic neck pain? A randomised controlled trial," Physiotherapy, 2015. 